\title{
Identification of the Retrograde Circuit in Left Posterior Fascicular Ventricular Tachycardia as a Target for Ablation
}

\author{
Adil Mahmood, ${ }^{1}$ Paramdeep Dhillon² and Peter Kabunga ${ }^{1,2}$ \\ 1. Department of Cardiology, Darent Valley Hospital, Dartford, Kent, UK; 2. King's College Hospital, London, UK
}

DOI: https://doi.org/10.17925/EJAE.2020.6.1.39

$\mathrm{S}$ low entry of calcium in partially depolarized Purkinje fibres is thought to mediate the genesis of fascicular ventricular tachycardia (VT). Verapamil reverses this influx and is effective at terminating VT. However, the efficacy of verapamil is variable and it may not be well tolerated due to side-effects, as seen in the case presented here. Idiopathic left posterior fascicular VT (ILPFVT) can be treated by localised catheter ablation in the left ventricular septum with a high success rate. Ablating at sites with the earliest retrograde-Purkinje potential (retro-PP) can be used for guiding successful ablation. In the case presented here, ablation at a site with a late retro-PP resulted in a successful ablation. This indicates that ablation at any site with retro-PP along the left ventricular posterior fascicular network may be sufficient in disrupting the ILPFVT circuit.

\section{Keywords}

Idiopathic left posterior fascicular ventricular tachycardia, verapamil, ablation

Disclosures: Adil Mahmood, Paramdeep Dhillon and Peter Kabunga have no financia or non-financial relationships or activities to declare in relation to this article.

Review Process: Double-blind peer review.

Compliance with Ethics: Informed consent was received from the patient involved in this case study.

Authorship: The named authors meet the International Committee of Medical Journal Editors (ICMJE) criteria

for authorship of this manuscript, take responsibility for the integrity of the work as a whole, and have given final approval for the version to be published.

Access: This article is freely accessible at touchCARDIO.com @ Touch Medical Media 2020

Received: 13 November 2019

Accepted: 2 December 2019

Published Online: 7 January 2020

Citation: European Journal of Arrhythmia \& Electrophysiology. 2020;6(1):39-42

Corresponding Author: Peter Kabunga, Department of Cardiology, Darent Valley Hospital, Darenth Wood Road, Dartford, Kent, UK, DA2 8DA. E:p.kabunga@nhs.net

Support: No funding was received in the publication of this article.
Idiopathic left posterior fascicular ventricular tachycardia (ILPFVT) is rare form of ventricular tachycardia (VT) that is characterised by right bundle-branch block (RBBB) morphology and superior axis in the presence of a structurally normal heart. ${ }^{1-3}$ The tachycardia is exquisitely sensitive to intravenous verapamil, ${ }^{1}$ and predominantly affects young adults. ${ }^{2-4}$ The most common symptoms include palpitations, presyncope, syncope and, less frequently, symptoms of congestive cardiac failure due to the development of a tachycardia cardiomyopathy. Ouyang et al. have reported mapping and successful ablation in ILPFVT by targeting the earliest retrograde-Purkinje potentials (retro-PPs) during sinus rhythm. ${ }^{5}$

Here we report a case of ILPFVT successfully treated with catheter ablation at a site with a late retro-PP.

\section{Case report}

A 40-year-old woman was referred to the cardiology clinic, following an abnormal electrocardiogram (ECG) at a pre-operative clinic for an elective cholecystectomy. She denied cardiac symptoms, but her ECG showed a broad complex rhythm with right bundle branch morphology and a superior axis/left axis deviation (Figure 1) consistent with a diagnosis of ILPFVT. Her cardiovascular examination and baseline investigations, including electrolytes and transthoracic echocardiogram, were normal. Oral verapamil $60 \mathrm{mg}$ three-times daily was initiated and within 12 hours sinus rhythm with only occasional premature ventricular contractions was seen. She declined to have further cardiac investigations including cardiac magnetic resonance imaging.

She remained in sinus rhythm at a 3-month follow-up but re-presented to the cardiac clinic 6 months later with intolerable side-effects from the verapamil. The ECG at the clinic revealed a recurrence of VT. An electrophysiology study was therefore performed due to recurrence of ILPFVT and a reluctance to try alternative medical therapy. The electrophysiology study, which was performed with minimal sedation, confirmed VT. Electro-anatomical mapping (Carto ${ }^{\circledR}$ 3, Biosense Webster, Irvine, CA, USA) of the left ventricle was performed using a retro-aortic approach, but catheter manipulation inadvertently terminated the VT at the start of the procedure. Despite repeated programmed stimulation with and without isoproterenol, sustained VT could not be reinitiated.

Mapping along the posterior mid to distal septal area of the left ventricle was performed. Sharp, high-frequency, low-amplitude potentials, suggestive of retrograde activation within the Purkinje network, were identified at the mid-inferior septum. Extensive mapping at this site revealed early (Figure 2) and late retro-PPs (Figure 3). A decision was made to ablate at a site with the late retro-PPs, as this site revealed the best pace-map with the clinical arrhythmia. At the onset of radiofrequency applications (irrigated $35-40 \mathrm{~W}, 50^{\circ} \mathrm{C}$ ), premature ventricular contraction automaticity, matching the clinical template, was seen (Figure 4). 
Figure 1: Baseline electrocardiogram showing a broad complex rhythm with a right bundle branch morphology and superior axis

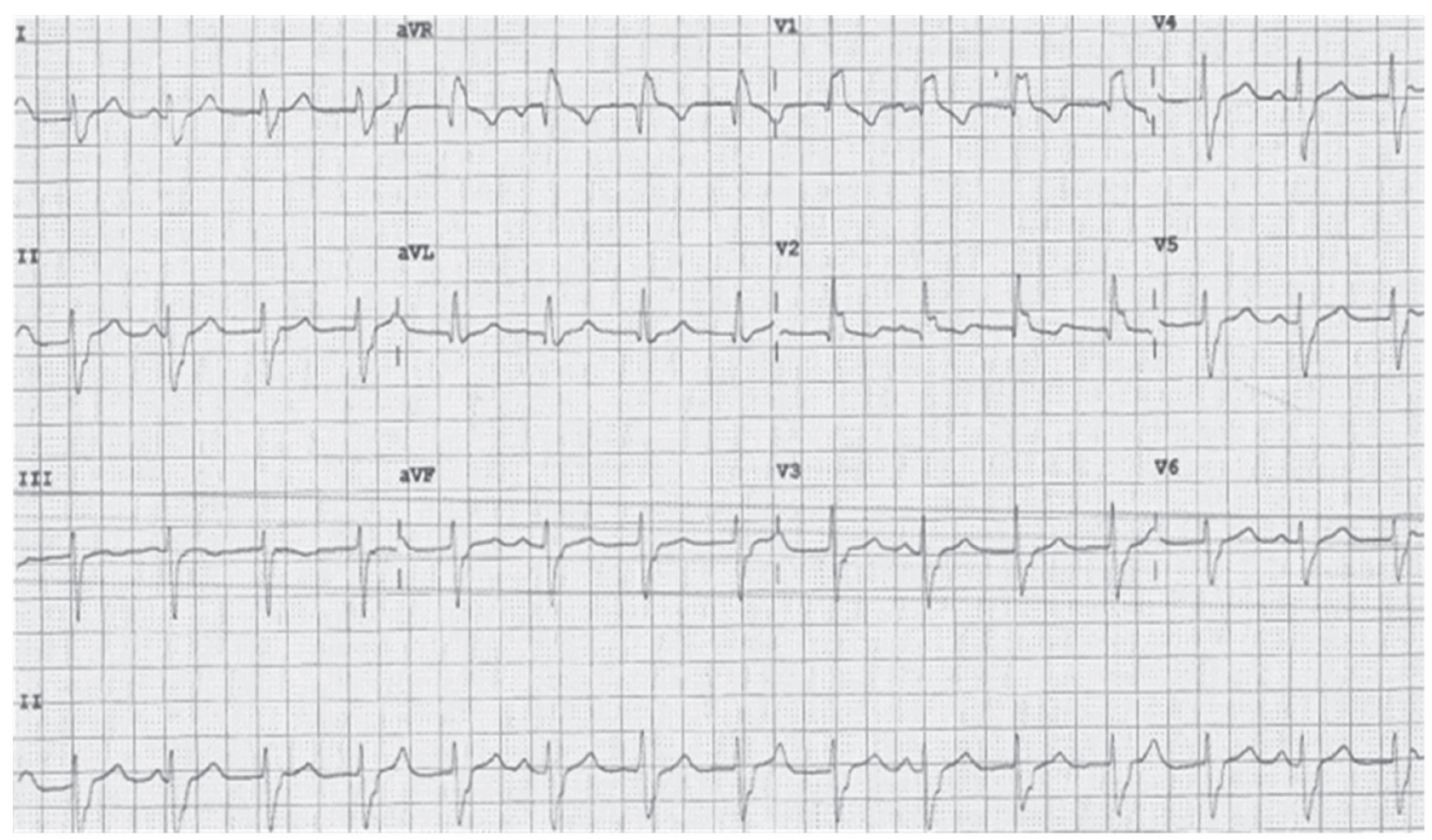

Figure 2: Mapping revealing early retrograde-Purkinje potentials in sinus rhythm

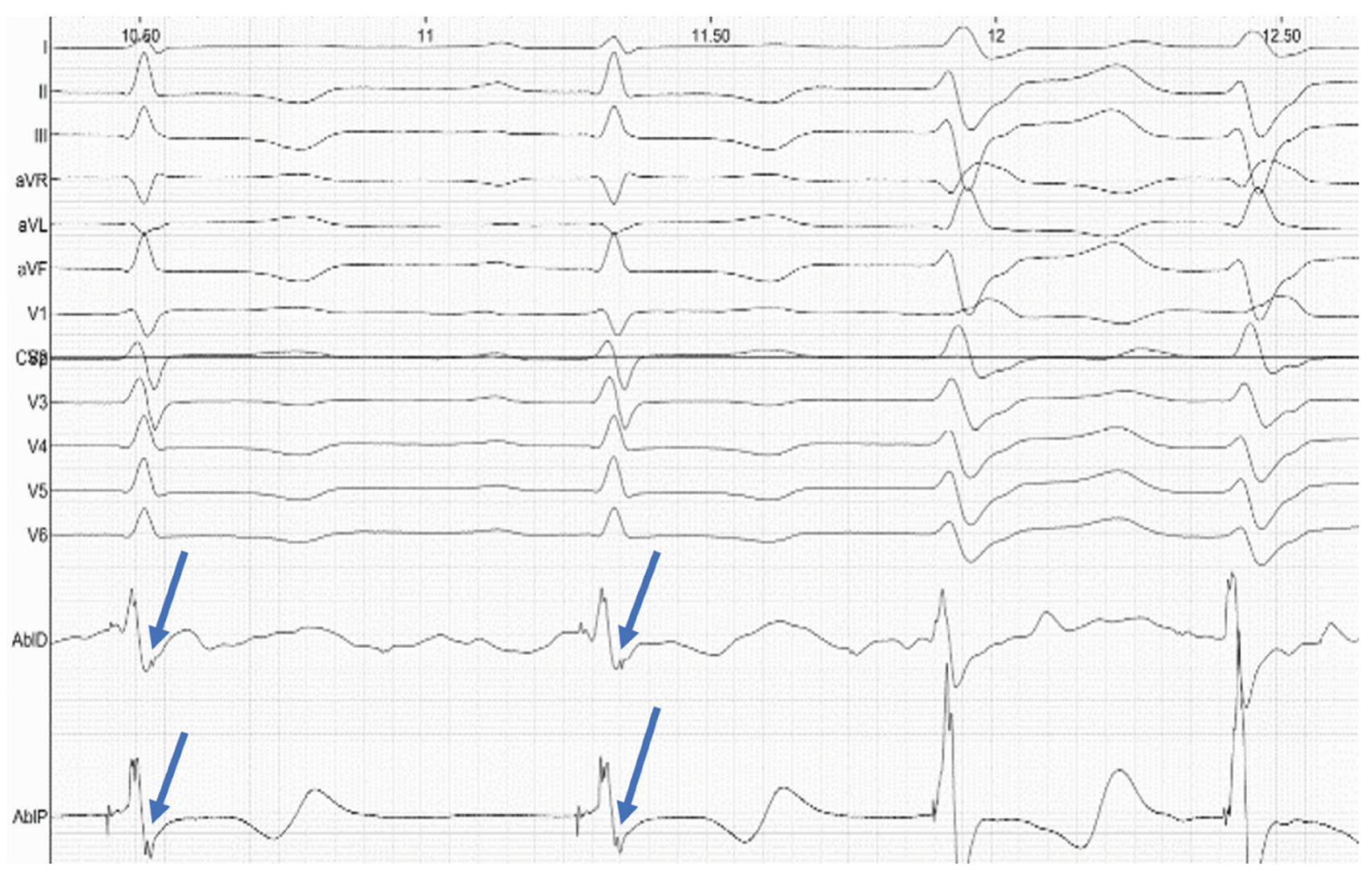


Figure 3: Mapping revealing late retrograde-Purkinje potentials in sinus rhythm

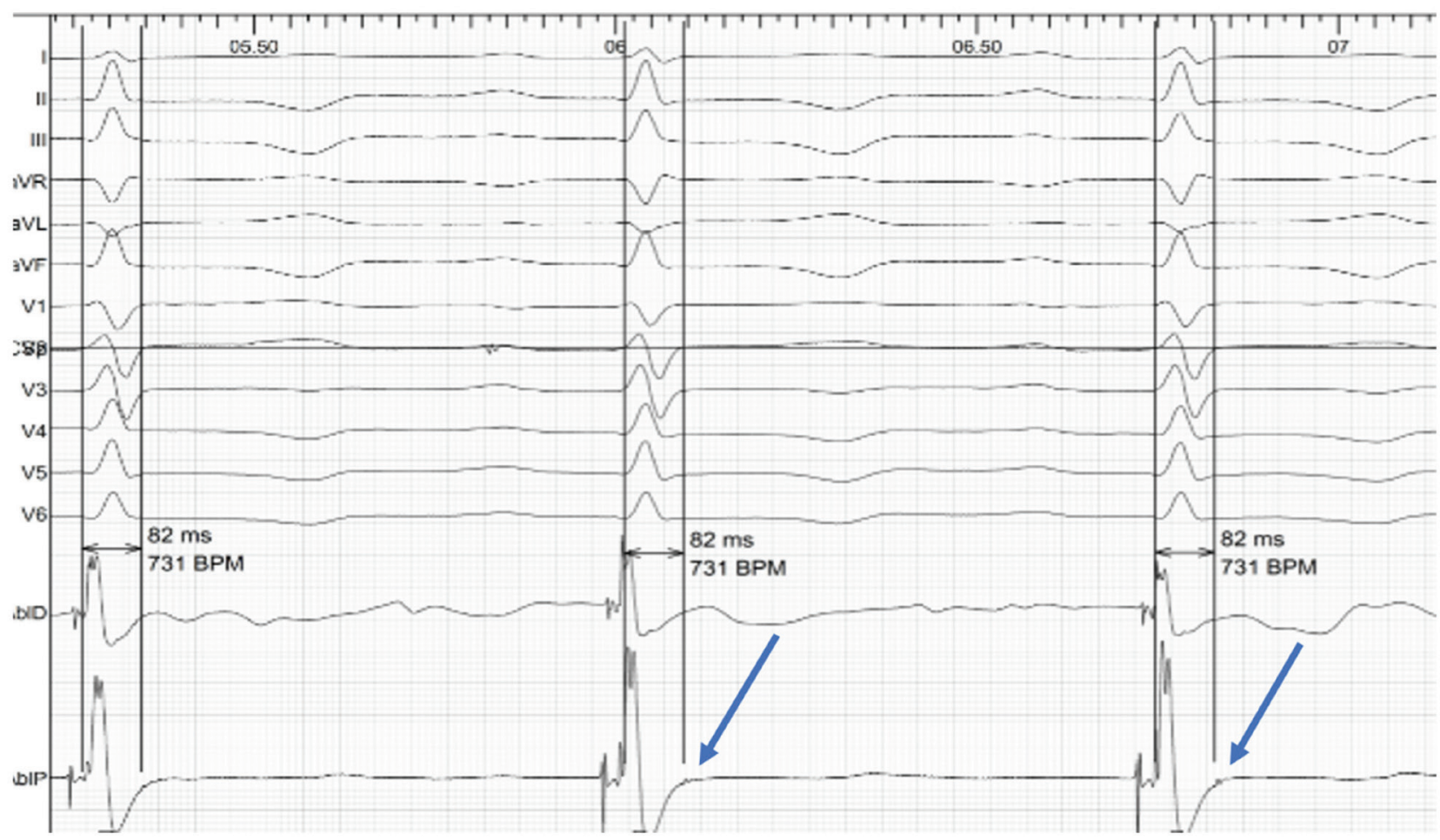

Late retrograde-Purkinje potentials in sinus rhythm marked with blue arrows.

Figure 4: Ablation at successful site with automaticity matching the clinical ventricular tachycardia

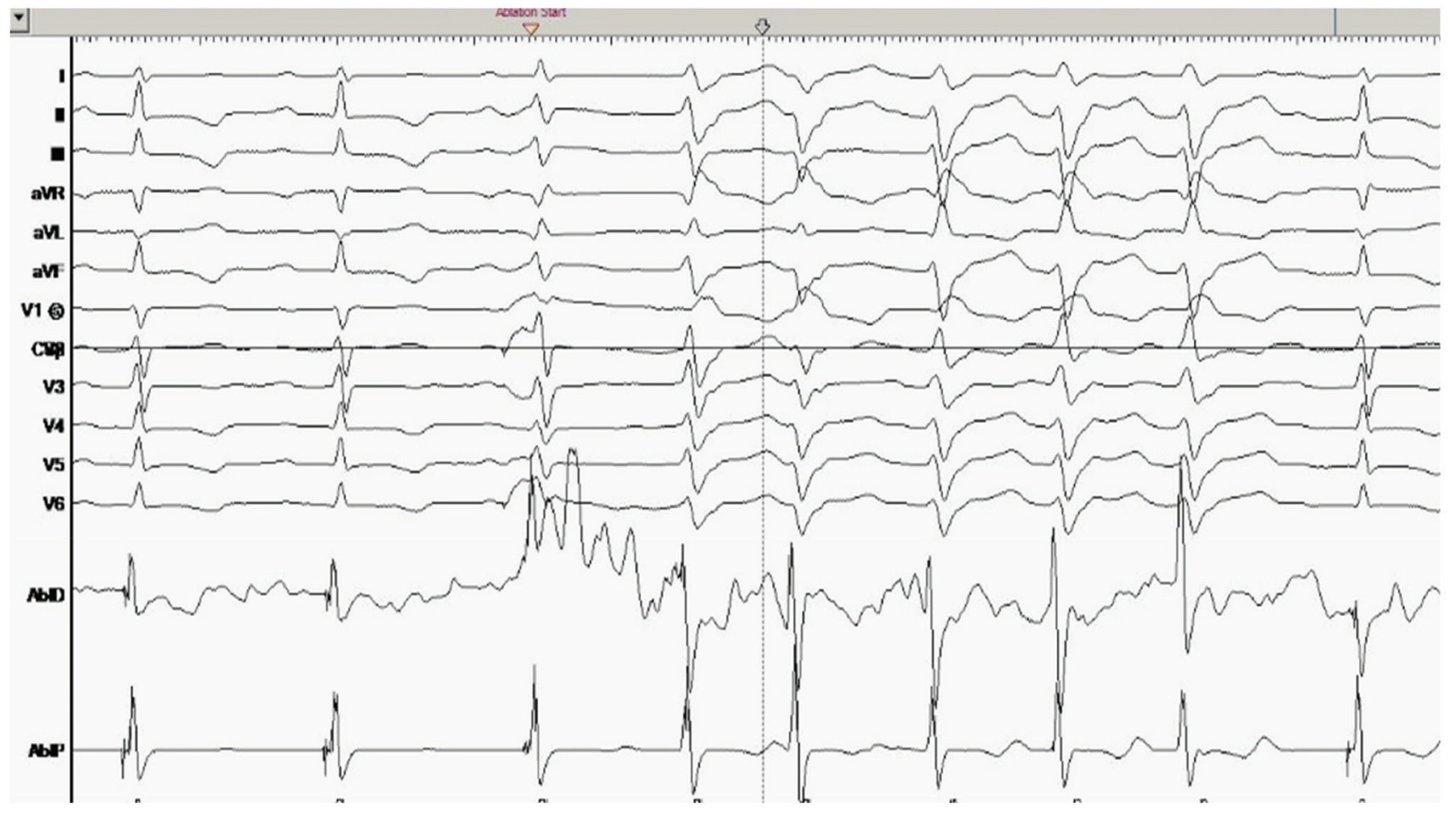

Following ablation, no premature ventricular contractions or ventricular arrhythmias were observed during the rest of the electrophysiology study or on telemetry over the ensuing 24 hours. The patient remains arrhythmia-free and off medications at 21 months follow-up.

\section{Discussion}

Slow entry of calcium in partially depolarized Purkinje fibres is thought to mediate the genesis of fascicular VT. Verapamil reverses this influx and is effective at terminating VT. ${ }^{1}$ However, the efficacy of verapamil 
is variable and it may not be well tolerated due to side-effects, as was seen in our patient. There are three forms of idiopathic fascicular VT. The most frequent form is ILPFVT followed by left anterior fascicular VT (RBBB with left axis deviation on ECG) and the least common form is upper septal fascicular VT (RBBB with normal axis or left bundle branch block or normal QRS with normal axis on ECG). ${ }^{3}$ ILPFVT can be treated by localised catheter ablation in the left ventricular septum with high success rate. ${ }^{6,7}$

Mid-diastolic potentials or earliest presystolic PPs are targeted during VT ablation. ${ }^{8}$ However, non-inducibility or inadvertent termination during catheter manipulation, as seen in our patient, often precludes successful ablation during tachycardia. Ablating at sites with the earliest retro-PP can be used for guiding successful ablation.
Previous studies have suggested that the VT circuit is confined to the posterior Purkinje system with an excitable gap and an area of slow conduction. Sites with the earliest retro-PP in sinus rhythm may represent retrograde Purkinje activation with slow conduction over a Purkinje-Purkinje, or alternatively Purkinje-ventricular-Purkinje connection with unidirectional block. ${ }^{8}$ In our patient, mapping at the mid-inferior septum along the course of the posterior fascicular network revealed several sites with both early and late retro-PPs. In the case presented here, ablation at a site with a late retro-PP resulted in a successful ablation.

The present case indicates that ablation at any site with retro-PP along the left ventricular posterior fascicular network may be sufficient in disrupting the ILPFVT circuit. $\square$
1. Belhassen $B$, Rotmensch $\mathrm{HH}$, Laniado S. Response of recurrent sustained ventricular tachycardia to verapamil. Br Heart $J$. 1981:46:679-82.

2. Ohe T, Shimomura K, Aihara N, et al. Idiopathic sustained left ventricular tachycardia: clinical and electrophysiologic characteristics. Circulation. 1988;77:560-8.

3. Gaita F, Giusteto C, Leclerc JF, et al. Idiopathic verapamil-responsive left ventricular tachycardia: clinical characteristics and long-term follow-up of 33 patients. Eur Heart J. 1994;15:1252-60.
4. Nakagawa $\mathrm{H}$, Beckman $\mathrm{KJ}$, McClelland $\mathrm{JH}$, et al, Radiofrequency catheter ablation of idiopathic left Radiofrequency catheter ablation of idiopathic left
ventricular tachycardia guided by a Purkinje potential. ventricular tachycardia guided
Circulation. 1993:88:2607-17.

5. Ouyang F, Cappato R, Ernst S, et al. Electroanatomic substrate of idiopathic left ventricular tachycardia. Unidirectional block and macroreentry within the Purkinje network. Circulation. 2002;105:462-9.

6. Shimoike E, Ueda N, Maruyama T, et al. Radiofrequency catheter ablation of upper septal idiopathic left ventricular tachycardia exhibiting left bundle branch block morphology. J Cardiovasc Electrophysiol. 2000:11:203-7.

7. Liu Y, Fang Z, Yang B, et al. Catheter ablation of fascicular ventricular tachycardia: long-term clinical outcomes and mechanisms of recurrence. Circ Arrhythm Electrophysiol 2015;8:1443-51.

8. Nogami A, Naito S, Tada H, et al. Demonstration of diastolic and presystolic Purkinje potentials as critical potentials in a macroreentry circuit of verapamil-sensitive idiopathic left ventricular tachycardia. J Am Coll Cardiol. 2000;36:811-23. 